\title{
MAX-DOAS measurements of formaldehyde in the Po-Valley
}

\author{
A. Heckel ${ }^{1}$, A. Richter ${ }^{1}$, T. Tarsu ${ }^{1}$, F. Wittrock ${ }^{1}$, C. Hak ${ }^{2}$, I. Pundt ${ }^{2}$, W. Junkermann ${ }^{3}$, and J. P. Burrows ${ }^{1}$ \\ ${ }^{1}$ Institute of Environmental Physics (IUP), P.O. Box 3304 40, University Bremen, 28334 Bremen, Germany \\ ${ }^{2}$ IUP, Im Neuenheimer Feld 229, University Heidelberg, 69120 Heidelberg, Germany \\ ${ }^{3}$ IMK-IFU, Kreuzeckbahnstrasse 19, 82467 Garmisch-Partenkirchen, Germany
}

Received: 14 January 2004 - Published in Atmos. Chem. Phys. Discuss.: 18 February 2004

Revised: 18 February 2005 - Accepted: 7 March 2005 - Published: 21 March 2005

\begin{abstract}
During the first measurement campaign of the EU FORMAT project in summer 2002 near Milan, northern Italy, ground-based scattered light differential optical absorption spectroscopy (DOAS) measurements were performed using a new multi-axis instrument. From the data set of this four week measurement period, the detailed analysis results of three days, 12-14 August, are presented exemplary. Slant column densities for formaldehyde ( $\mathrm{HCHO}$ ) and the oxygen dimer $\left(\mathrm{O}_{4}\right)$ have been retrieved, employing fitting windows from $335 \mathrm{~nm}$ to $357 \mathrm{~nm}$ and $350 \mathrm{~nm}$ to $360 \mathrm{~nm}$ respectively. In order to convert slant into vertical columns radiative transfer calculations were perfomred using aerosol parameters derived from the actual $\mathrm{O}_{4}$ measurements. By analysing the measurements from different viewing directions (zenith, $4 \mathrm{x}$ off-axis) vertical profile information, and in particular mixing ratios for the boundary layer have been derived for the first time for $\mathrm{HCHO}$ with a multi-axis DOAS (MAX-DOAS) instrument. HCHO vertical columns are in the range of 5 to $20 \cdot 10^{15} \mathrm{molec} / \mathrm{cm}^{2}$ with an relative error of about $15 \%$. This corresponds to $\mathrm{HCHO}$ mixing ratios in the boundary layer of $0.7 \mathrm{ppb}$ to $4.2 \mathrm{ppb}$, which is in excellent agreement with simultaneous measurements from both a Hantzsch in-situ and a long-path DOAS instrument operated at the same place.
\end{abstract}

\section{Introduction}

In spite of all efforts towards emission reduction, photochemical smog and as a result enhanced levels of tropospheric ozone are a widespread problem in highly populated areas and also in regions where biomass burning takes place. Ozone formation is triggered by elevated concentrations of $\mathrm{NO}_{\mathrm{x}}$ and hydrocarbons, both from anthropogenic and nat-

Correspondence to: A. Richter

(andreas.richter@iup.physik.uni-bremen.de) ural sources. Formaldehyde is the most abundant carbonyl in the atmosphere, and is one intermediate product of the basic oxidation cycles of hydrocarbons. Besides its role in the processes characterising photochemical smog, $\mathrm{HCHO}$ is of particular importance as its concentrations are mainly determined by the abundances of other hydrocarbons, and can therefore be used as an indicator for volatile organic compounds (VOC) (Finlayson-Pitts and Pitts, 2000). In contrast to other hydrocarbons, HCHO concentrations can be measured using remote sensing in the UV/visible spectral region, both from the ground as in this study and from space using e.g. the Global Ozone Monitoring Experiment (GOME, Burrows et al., 1999; Palmer et al., 2001), SCIAMACHY, the Scanning Imaging Absorption Spectrometer for Atmospheric Cartography (Bovensmann et al., 1999) or in the future OMI, the Ozone Monitoring Instrument.

The FORMAT (Formaldehyde as a tracer of photo oxidation in the Troposphere) project is a EU funded three-year project focused on measuring, modelling and interpreting $\mathrm{HCHO}$ in the heavily polluted region of the Po-Valley in Northern Italy. Its single most important objective is to improve techniques, which are used to measure formaldehyde. Therefore two large measurement campaigns, one in summer 2002 and a second in autumn 2003, constitute a mayor part of the total activities.

During these campaigns, ground-based measurements at three locations (close to Voghera, in Bresso (Milan) and in Alzate, see Fig. 1) as well as airborne measurements were performed, using a wide range of different in-situ and remote sensing measurement techniques. With this arrangement, air masses in close vicinity to the major source (Bresso, Milan) of pollution as well as those up to $50 \mathrm{~km}$ away from the source region, in a more rural environment (Alzate, Voghera) are examined. Depending on wind conditions the latter locations can be influenced by the Milan pollution plume. In the beginning of the summer campaign 2002 inter-comparison measurements were performed in Alzate

(C) 2005 Author(s). This work is licensed under a Creative Commons License. 


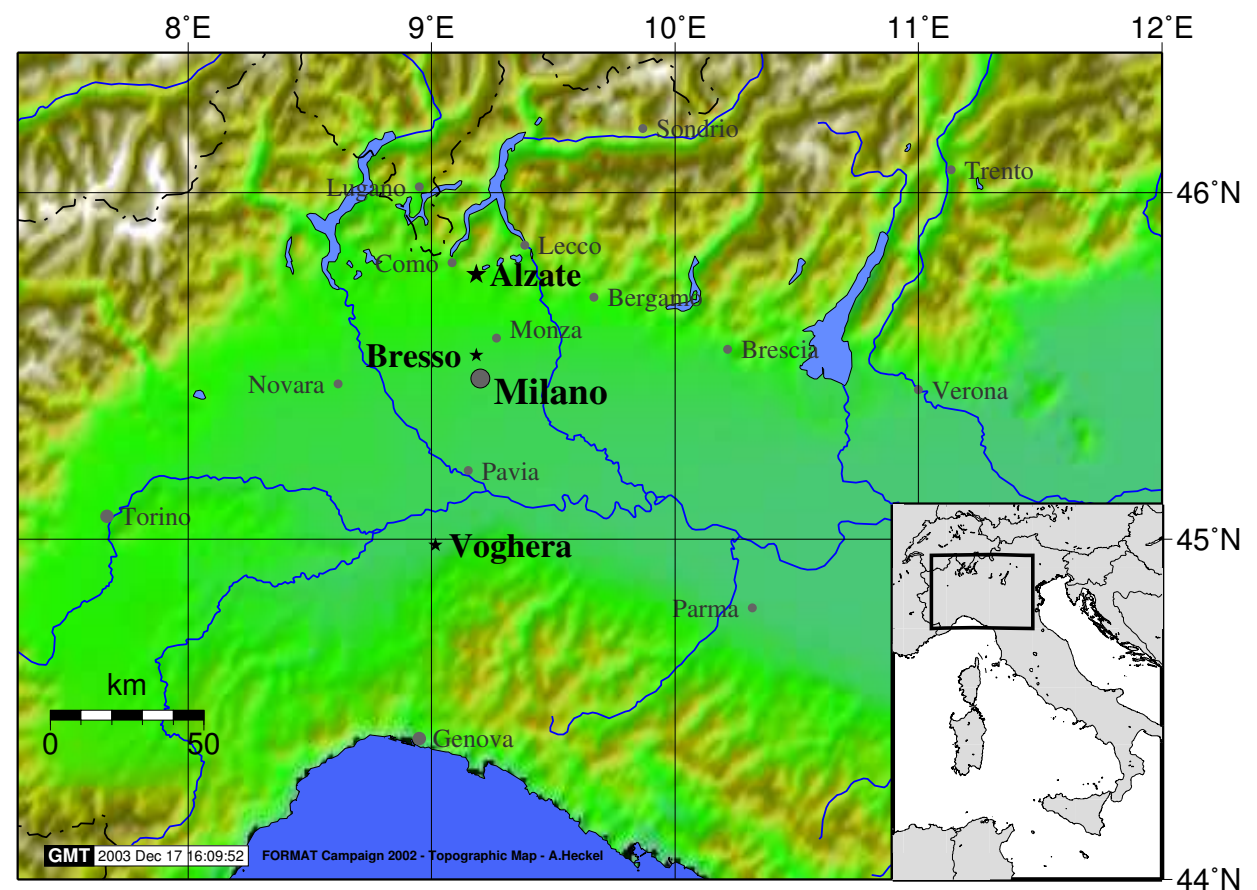

Fig. 1. Map of the measurement area, showing the Po-Valley in northern Italy. The location of the measurements presented here was Alzate, $50 \mathrm{~km}$ north of Milan. Within the frame of the FORMAT campaign 2002 other instruments were placed in Bresso and close to Voghera.

employing all multi-axis- and long-path DOAS instruments involved in the campaign.

In this article, results from ground-based UV/VIS measurements of $\mathrm{HCHO}$ recorded during the first campaign in July and August 2002 are reported. The MAX-DOAS instrument was situated in Alzate $\left(9.183^{\circ} \mathrm{N}, 45.767^{\circ} \mathrm{E}\right)$. Usually, southerly wind conditions are prevailing and the Milan plume is extending over Alzate, while during North Foehn conditions very dry and clean air is coming down from the alps.

First, the instrumental set-up and the measurement technique used are briefly described, followed by a discussion of the analysis procedure, in particular with respect to the retrieval of vertical columns and profile information. In Sects. 4 and 5, HCHO results are presented for some selected days and compared to independent measurements, followed by a discussion of errors.

\section{Instrument}

The instrument deployed, is a standard UV/VIS DOAS instrument similar to those used for many atmospheric measurements. A detailed description of the instrument and the telescope system can be found in Wittrock et al. (2004). Its main components are a telescope and a grating spectrometer with a CCD detector controlled by a PC. The spectrometer is of the Czerny-Turner type and provides a spectral resolution of $0.5 \mathrm{~nm}$ FWHM, derived from a representative line of a mercury-cadmium spectrum. The measured wavelength interval ranges from $312 \mathrm{~nm}$ to $396 \mathrm{~nm}$.

The telescope has a rotating mirror to select the elevation of the viewing direction and is pointed to a fixed azimuth. Due to the design of the telescope, measurements in the zenith and between $0^{\circ}$ and $30^{\circ}$ elevation are possible.

Measurements in directions with different elevation angles are taken consecutively but within a short time period to avoid complications due to changes in the atmospheric composition during a measurement cycle. The data acquisition software enables entirely autonomous measurements and to choose up to four single off-axis directions plus the zenith view. The sketch in Fig. 2 depicts the chosen geometry with the elevation angles $3^{\circ}, 6^{\circ}, 10^{\circ}$ and $18^{\circ}$ as well as the basic pathway through certain atmospheric layers considering single scattering. The off-axis directions have similar stratospheric light paths but for decreasing elevations the path length in the lower troposphere is increasing. This leads to a large enhancement in absorption, with respect to the zenith geometry, for species with high concentrations in the boundary layer, as, for example, formaldehyde.

During a measurement cycle, data were acquired at each elevation angle for about $60 \mathrm{~s}$, averaging over 2 to 26 scans depending on illumination, to improve the signal to noise ratio. Hence a full cycle of measurements in all five directions lasted $5 \mathrm{~min}$, which is also the time resolution of the data set.

In total, continuous measurements were taken for 30 days. During the inter-comparison period in the beginning of the campaign, from 21 July to 26 July, the off-axis port of the 
instrument was pointing towards the west. Afterwards, until the end of measurements on 19 August, the instrument was turned, looking SSW. This set-up was chosen to prevent direct sunlight from entering the off-axis port of the instrument during sunset, thereby facilitating off-axis measurements during the entire day.

Similar instruments have been used in the recent past for tropospheric measurements of $\mathrm{BrO}$ (Hönninger and Platt, 2002; Leser et al., 2003), $\mathrm{SO}_{2}$ (Bobrowski et al., 2003) and $\mathrm{NO}_{2}$ (Wittrock et al., 2004), and using only one off-axis viewing direction also for HCHO (Roozendael et al., 2003). Here we report on the first multi-axis measurements focused on HCHO using several off-axis measurements to retrieve profile information.

\section{Data analysis}

The data analysis is divided into several steps, which are described in some detail below. In a first step the amount of $\mathrm{HCHO}$ is determined separately at each elevation angle using the DOAS technique (Solomon et al., 1987; Platt, 1994). These HCHO slant column densities depend on the viewing geometry, the vertical profile of $\mathrm{HCHO}$ and also surface albedo and aerosol loading. In a second step, the measurements are simulated by a radiative transfer model (SCIATRAN, Rozanov et al. (2001)) and the vertical profile of HCHO is varied until a consistent solution for all viewing directions is obtained. Prior to that, a realistic aerosol parameterisation has to be found. This is achieved by varying the aerosol settings of the model until the measurements of the oxygen dimer $\mathrm{O}_{4}$, recorded at the same time, can be reproduced. Up to this point, the $\mathrm{HCHO}$ vertical profile is only determined by its relative shape, not by absolute values. Therefore, in the last step, the assumed vertical profile of HCHO will be scaled with the retrieved vertical column, enabling finally the determination of the volume mixing ratios (VMR) of $\mathrm{HCHO}$ at surface level using the profile value corresponding to the lower-most layer.

\subsection{DOAS retrieval}

From the spectra recorded by the instrument, different trace species with suitable band structures in their absorption cross-sections can be retrieved applying the Differential Optical Absorption Spectroscopy (DOAS) technique. Under the assumption of an optically thin atmosphere, the LambertBeer law can be applied. By forming the ratio of the logarithm of two spectra, the extinction term can be obtained. The absorption by the atmospheric trace gases contributes to this term as well as scattering processes. To account for the latter, a polynomial of low order is fitted and subtracted from the extinction.

Ideally, an absorption free measurement should be used as a background spectrum for the ratio. In the absence of such

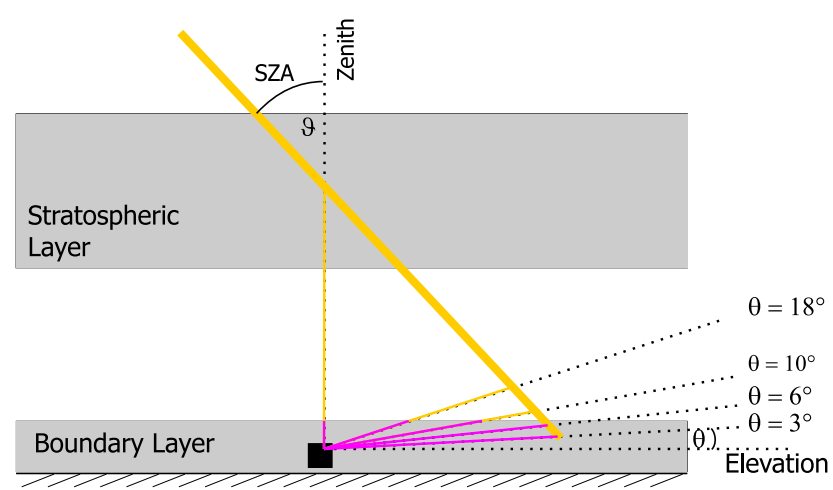

Fig. 2. Sketch of the viewing geometry of the instrument. Explicitly, the enhancement of absorption path length in the boundary layer due to different off-axis viewing directions close to the horizon can be seen.

a measurement a zenith spectrum can be used as background spectrum, assuming a horizontally homogenous atmosphere. This spectrum should be taken at a small solar zenith angle to minimise absorption and thereby maximising the differential signal. However, this background absorption has to be taken into account later in the analysis. In this study, one single background was used for the entire time series, which has the advantage of producing comparable results within this period. To keep the influence of the amount of trace gas in the background spectrum minimal, a noon zenith spectrum from a day with a very small slant column density was used, namely 7 August 2002.

The quantity retrieved with this method is a so called differential slant column density (DSCD), which is the difference of the column amount of the absorber integrated along the light path through the atmosphere and the absorber amount in the background spectrum.

For the formaldehyde retrieval a fitting window from $335 \mathrm{~nm}$ to $357 \mathrm{~nm}$ is applied. This window contains three absorption bands at $353 \mathrm{~nm}, 343 \mathrm{~nm}$ and $339 \mathrm{~nm}$. These are vibrational transitions at the visible end of the energetically lowest (first) electronic transition $\left(\mathrm{n} \rightarrow \pi^{*}\right)$ around $303 \mathrm{~nm}$ (Dieke and Kistiakowsky, 1934). The fitted polynomial is of the degree 5 and absorptions by $\mathrm{O}_{3}, \mathrm{NO}_{2}$, the oxygen dimer $\mathrm{O}_{4}$ as well as $\mathrm{BrO}$ are taken into account. The effect of Raman scattering (Ring effect Grainger and Ring, 1962) is compensated by fitting a synthetic Ring spectrum as an additional absorber. This Ring spectrum was computed by the radiative transfer model SCIATRAN (Vountas et al., 1998). Figure 3 shows a typical example for the fit quality in case of high $\mathrm{HCHO}$ absorptions along the light path.

The next analysis step is to convert slant into vertical columns by modelling the radiative transfer and computing the enhancement or so called air mass factors (AMF), which are defined as the ratio of slant to vertical column densities, $A M F=S C D / V C D$. In our analysis, the radiative transfer 


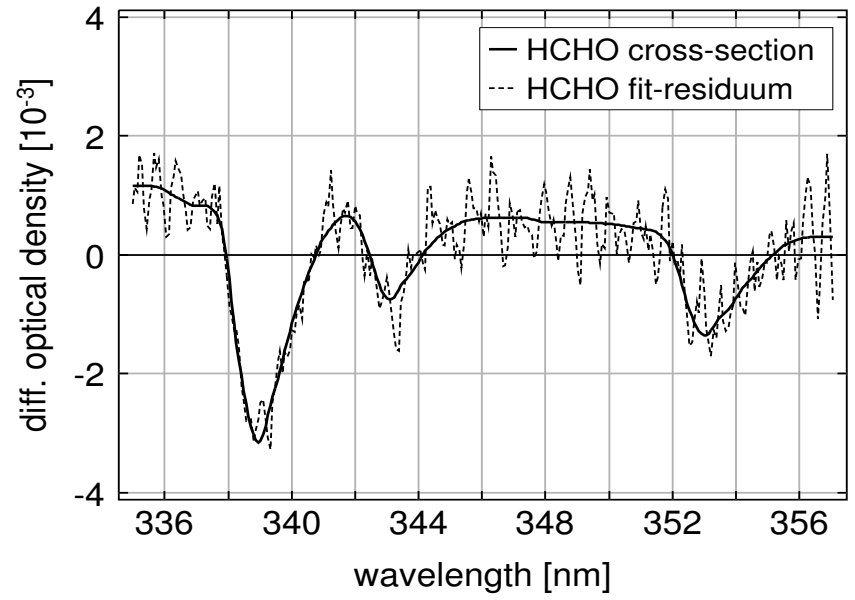

Fig. 3. HCHO differential absorption cross-section and fit-residual for 28 July 2002 , SZA: $51.11^{\circ}$, line of sight: $3^{\circ}$ elevation.

model SCIATRAN is used, which is able to compute AMFs not only for zenith but also for off-axis viewing directions. SCIATRAN calculates the radiation field for a spherical planetary atmosphere using a combined differential-integral approach involving the Picard iterative approximation (CDIPI) (Rozanov et al., 2001). Full multiple scattering is taken into account, the importance of which is discussed by Wittrock et al. (2004). As mentioned above, the absorption in the background spectrum also has to be considered in the calculations of the vertical column as follows

$\operatorname{VCD}_{\theta}=\frac{\operatorname{DSCD}(\varphi, \vartheta, \theta)+\operatorname{SCD}_{\mathrm{bg}}\left(\varphi_{\mathrm{bg}}, \vartheta_{\mathrm{bg}}, \theta_{\mathrm{bg}}\right)}{\operatorname{AMF}(\varphi, \vartheta, \theta)}$

Where DSCD are the retrieved differential slant column densities, $\mathrm{SCD}_{\mathrm{bg}}$ stands for the slant column density of the background spectrum which in this case is the zenith spectrum at the lowest solar zenith angle of 7 August 2002 (at 10:54 UTC, $30.24^{\circ}$ SZA). Slant column densities and AMFs depend on the solar zenith angle $\vartheta$, the relative azimuth angle $\varphi$ between sun and viewing direction and on the elevation angle $\theta$ of the viewing direction. The resulting vertical column densities, retrieved from the different viewing directions, are independent measurements of the same quantity: the true vertical column, and hence provide more information than measurements obtained from the traditionally used instruments with only one fixed zenith viewing direction. Explicitly the difference between the retrieved VCDs, which ideally should vanish, provides a verification of the quality of the AMFs and implicitly verifies the applied model parameters. This improves the VCD retrieval and can provide profile information.

To determine the amount of absorption in the background spectrum, the same approach is used assuming that the temporal change of trace gas concentrations can be neglected within a narrow time interval around the background spectrum:

$$
\operatorname{VCD}_{\theta}=\frac{\operatorname{DSCD}\left(\varphi_{\mathrm{bg}}, \vartheta_{\mathrm{bg}}, \theta\right)}{\operatorname{AMF}\left(\varphi_{\mathrm{bg}}, \vartheta_{\mathrm{bg}}, \theta\right)-\operatorname{AMF}\left(\varphi_{\mathrm{bg}}, \vartheta_{\mathrm{bg}}, \theta_{\mathrm{bg}}\right)}
$$

Using Eq. (2), a background vertical column can be calculated from each off-axis viewing direction, and with the appropriate set of AMF the DSCD from the four viewing directions yield one vertical column. How this set of AMFs is derived will be described in the next section. Finally, multiplying the retrieved $\mathrm{VCD}_{\mathrm{bg}}$ with the $\mathrm{AMF}_{\mathrm{bg}}$ gives the required $S C D_{b g}$.

\subsection{Deriving profile information}

If the atmosphere is assumed to be horizontally homogeneous within the range of the measurement volume the same vertical column should be measured in all five viewing directions. As shown in Fig. 2, the light paths through the troposphere strongly depend on the elevation angle $\theta$. Due to different light paths through corresponding atmospheric layers the viewing directions have a different sensitivity towards absorption in different heights. Only by assuming an appropriate vertical profile of $\mathrm{HCHO}$ in the AMF calculations, all measurements will agree in the vertical column. The retrieval of profile information therefore is based on varying the a priori profile in the radiative transfer calculations until good agreement is found for a set of measurements.

In order to obtain such a suitable set of AMFs, the radiative transfer calculations were repeated, utilising different trace gas profiles until the retrieved vertical columns of the different viewing directions match best. Due to diurnal changes of the vertical profiles, the matching holds generally only for certain time periods. The length of these time intervals depends strongly on the dynamic situation of the atmosphere. In principle the same analysis has to be done for every measurement of the day employing an automated inversion. While this will be implemented in future work, only a few data points shall be presented in this study, in order to demonstrate the feasibility of this approach.

\subsection{Determining the aerosol settings}

As introduced above, the light path of the off-axis viewing directions is strongly dependent on aerosol extinction. In a clear atmosphere, the viewing directions with small elevation angles have very long light paths in the lowest atmospheric layers, and differences between viewing directions are large. In contrast, in a hazy atmosphere the visibility is reduced and the light path of the lowest viewing directions is limited by scattering, reducing the differences between the viewing directions. As these differences are used to derive information on the vertical profile of the absorber, a good estimate of the aerosol extinction profile is needed to facilitate profile retrieval. 


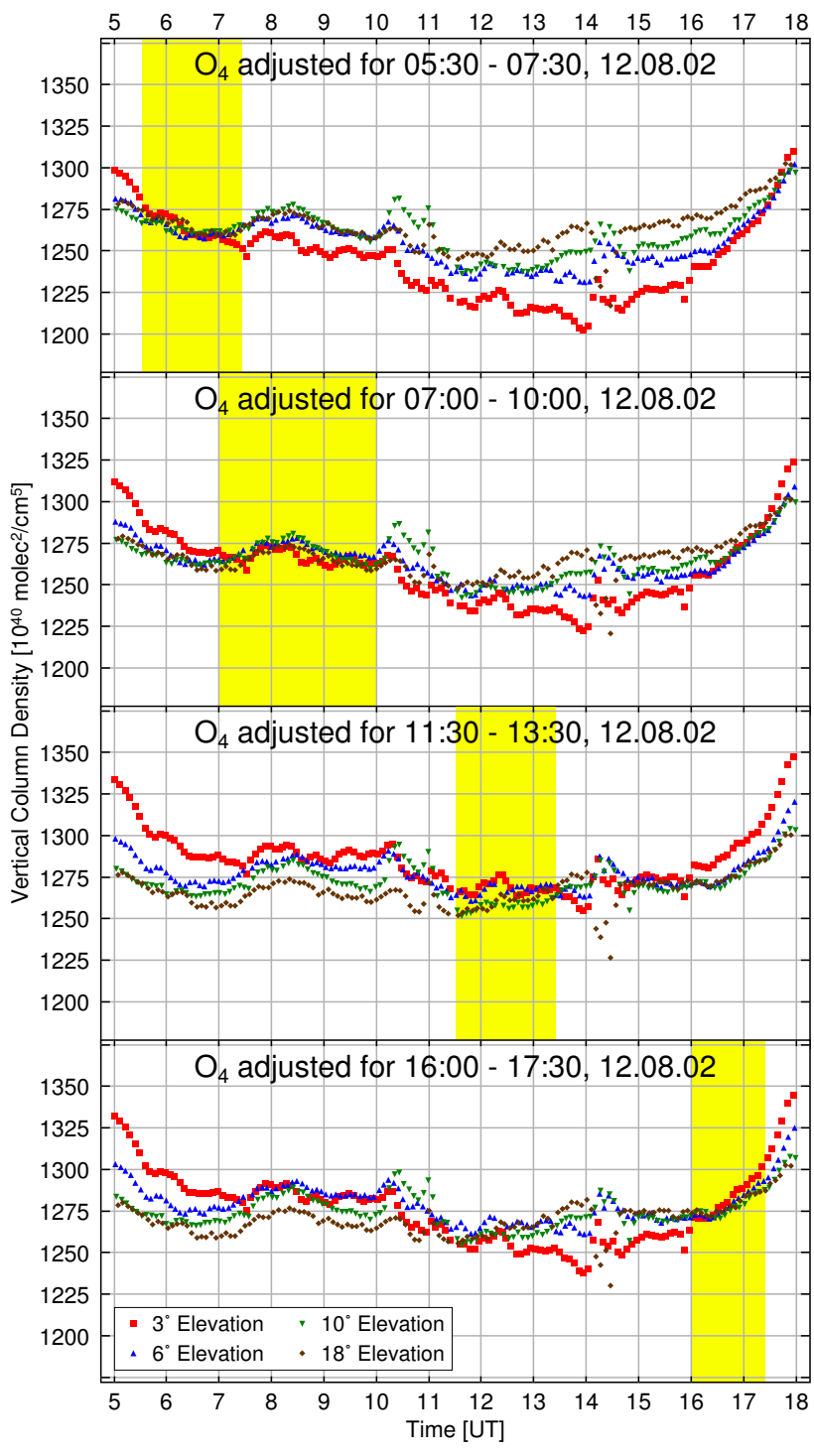

Fig. 4. Vertical columns of $\mathrm{O}_{4}$ on 12 August 2002, derived using four different aerosol settings while calculating the AMFs. The resulting AMFs are appropriate for different time periods, which are highlighted in yellow.

In order to estimate the aerosol extinction, measurements of the oxygen dimer $\mathrm{O}_{4}$ were analysed as already suggested by Wagner et al. (2002). As the VMR (volume mixing ratio) profile of the dimer varies with the square of the $\mathrm{O}_{2}$ monomer, the profile of $\mathrm{O}_{4}$ is well known, easily computed and mainly dependent on the pressure and temperature profile. Besides the profiles, important remaining parameters in the radiative transfer are albedo and those determining the aerosol extinction, such as aerosol composition, relative humidity or extinction profile. In analogy to the profile retrieval described above, albedo and mainly the aerosol extinction profile are varied until good agreement is found for the $\mathrm{O}_{4}$ vertical columns derived from all viewing directions. The

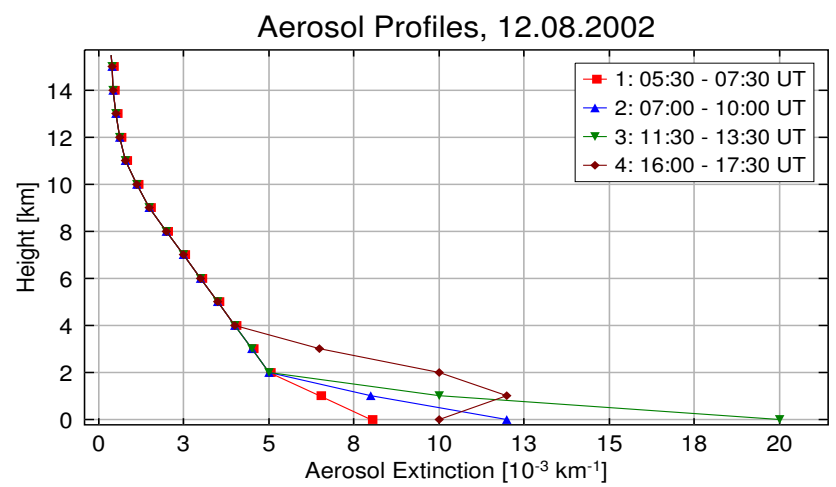

Fig. 5. Aerosol extinction profiles used for retrieval of $\mathrm{O}_{4}$ vertical columns at certain points in time on 12 August 2002.

settings derived in this manner are then used in the HCHO profile retrieval. The measurements, however, do not provide enough information for a full retrieval. Thus, assumptions or external data about the aerosol composition and information about temperature, pressure and humidity profiles are necessary.

To optimise the quality of the $\mathrm{O}_{4}$ fit, a wavelength window from $350 \mathrm{~nm}$ to $370 \mathrm{~nm}$ was chosen. In addition to $\mathrm{O}_{4}$, a synthetic Ring cross-section as well as $\mathrm{O}_{3}, \mathrm{NO}_{2}$ and $\mathrm{BrO}$ were considered in the analysis. A polynomial of 2 nd degree was subtracted.

As an example, vertical columns of $\mathrm{O}_{4}$ for 12 August are shown in Fig. 4. For different times of the day, different aerosol profiles have to be assumed, illustrating the diurnal variation of the aerosol extinction. Figure 5 presents the aerosol extinction profiles employed for the time periods indicated in Fig. 4. Although this day represents a very clear situation, the disturbances around 10:30 UT and 14:00 UT are caused by very thin partial cloud cover in some of the instrumental lines of sight. The absolute value of the retrieved $\mathrm{O}_{4}$ column agrees with the column derived from the pressure profile, which equals $1.26 \cdot 10^{43} \mathrm{molec}^{2} / \mathrm{cm}^{5}$, within an error range of $8 \%$. During the day the column varies about $\pm 2 \%$, which is in agreement with the expectation of a nearly constant $\mathrm{O}_{4}$ column.

\section{Results and discussion}

The analysis described above has been applied to measurements from selected days of the FORMAT campaign 2002. For the sake of simplicity the analysis was limited to days without cloud influence, namely 12 to 14 August 2002.

\subsection{Slant columns}

A closer look at the slant columns of $\mathrm{HCHO}$ and $\mathrm{O}_{4}$ (see Fig. 6) and a comparison with meteorological data allows a 


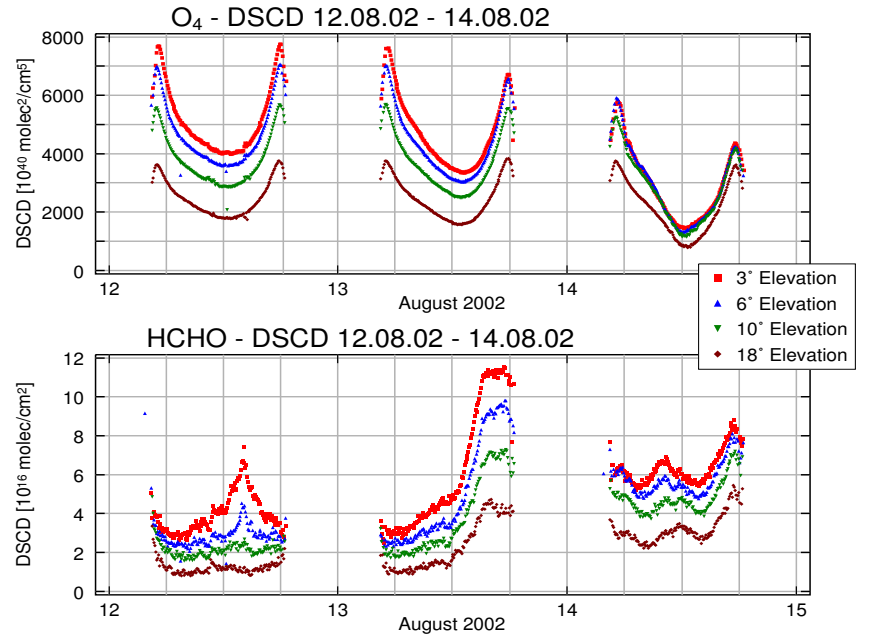

Fig. 6. Time series of slant columns of $\mathrm{O}_{4}$ and $\mathrm{HCHO}$ during 12 to 14 August. Starting with a very clean day, the atmosphere is becoming increasingly polluted, as can be seen from the $\mathrm{O}_{4}$ DSCD. The HCHO DSCD show an increase on 13 August followed by decreased values on the 14th. This is related to the strong increase in aerosol extinction (visible in the $\mathrm{O}_{4} \mathrm{DSCD}$ ), whereas the $\mathrm{HCHO}$ VCD was still increasing.

first characterisation of the conditions during these three days in August 2002.

The smooth behaviour of the $\mathrm{O}_{4}$ indicates that there were reasonably long time periods during the days without cloud influence. As one can see from Fig. 6 the slant columns, especially of the lower viewing directions are well separated on 12 August, as expected for a very clear day.

Meteorological data from a ground station at the measurement site show wind coming from northerly directions and a low relative humidity of about $50 \%$ or less during the day. This is typical for northerly Foehn wind conditions (Weber and Prévôt, 2002) thus one can easily identify these periods in the $\mathrm{O}_{4}$ measurements. The slight asymmetry of the $\mathrm{O}_{4}$ columns with respect to local noon is a result of the dependence of the AMF, and therefore the slant column, on the relative azimuth and depends on how the telescope is pointed to the sun (for more details see Wittrock et al., 2004).

On 13 August, $\mathrm{O}_{4}$ measurements from different elevation angles come closer together during the afternoon and on 14 August the differences between the measurements with elevations smaller than $10^{\circ}$ above the horizon almost vanish. This is evidence for the increasing aerosol amount during this period. The corresponding optical depth of the total aerosol extinction derived from the $\mathrm{O}_{4}$ analysis at $360 \mathrm{~nm}$ is increasing from about 0.05 on 12 August to $0.3-0.5$ on 14 August. Similar to clouds, the aerosol decreases the free path length of the photons. Thus, the light paths of photons from different elevation angles become similar and so become the slant columns.

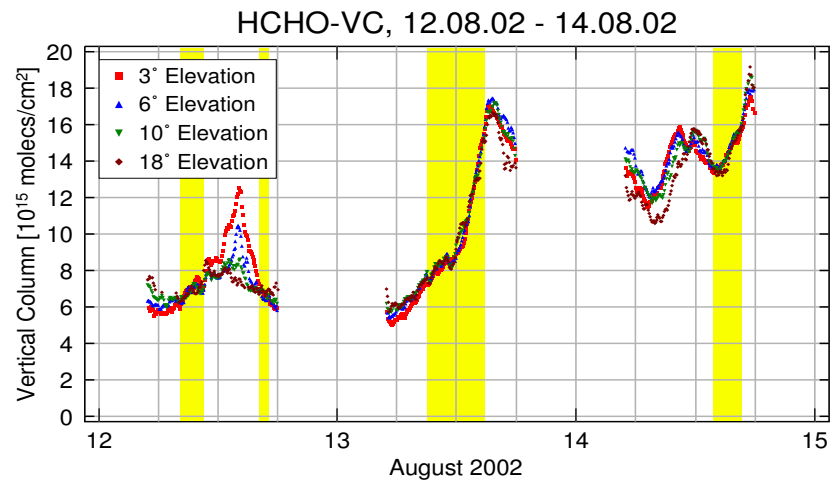

Fig. 7. Time series of vertical columns of $\mathrm{HCHO}$ from 12 to 14 August 2002. The columns were derived with only one AMF set per day, which fits best during the highlighted time periods.

From a meteorological point of view, the foehn is weakening during this time period. The wind direction is changing to south-easterly directions and the relative humidity is increasing during these two days. The southerly winds also bring more polluted air from the Po-Valley region (see Prévôt et al., 1997). Summarising, the North Foehn and also heavy rainfall before 12 August, which cleared the atmosphere, led to very clean conditions with extraordinary good visibility and low HCHO values on 12 August and in the beginning of 13 August. Later the atmosphere became polluted and hazy, decreasing the visibility particularly within the lower troposphere.

Looking at the development of HCHO DSCDs during the period, an overall increasing trend can be observed, at least for the directions with larger elevation angle. In contrast, the lower directions show a decrease from 13 to $14 \mathrm{Au}-$ gust. As will be shown later in this section, this decrease is mainly due to the increase of aerosol extinction and in fact high $\mathrm{HCHO}$ columns persisted on these days. Note, that the decrease in differences between the viewing directions observed for $\mathrm{O}_{4}$ on the second and third day is not as clear for $\mathrm{HCHO}$. This reflects the different vertical distributions of $\mathrm{O}_{4}$ and $\mathrm{HCHO}$. The latter is more concentrated in the lower layers, and while the $\mathrm{O}_{4}$ columns are nearly identical for the lowest viewing directions on 14 August, there still remains some profile information in the $\mathrm{HCHO}$ measurements.

During the time interval shown, two periods with significantly increased $\mathrm{HCHO}$ have to be pointed out: First, there was a large increase only for the lowest two viewing directions in the afternoon of 12 August. This increase cannot be explained by a change in light path since there is no similar pattern in the $\mathrm{O}_{4}$ at the same time. The only explanation is a local increase in $\mathrm{HCHO}$ close to the surface, probably by emission from a local source. The second increase, taking place on the afternoon of 13 August is detected in all four lines of sight simultaneously. The $\mathrm{O}_{4}$ columns show a slight decrease during this time period, indicating that the effective light path was not increasing but rather decreasing, 


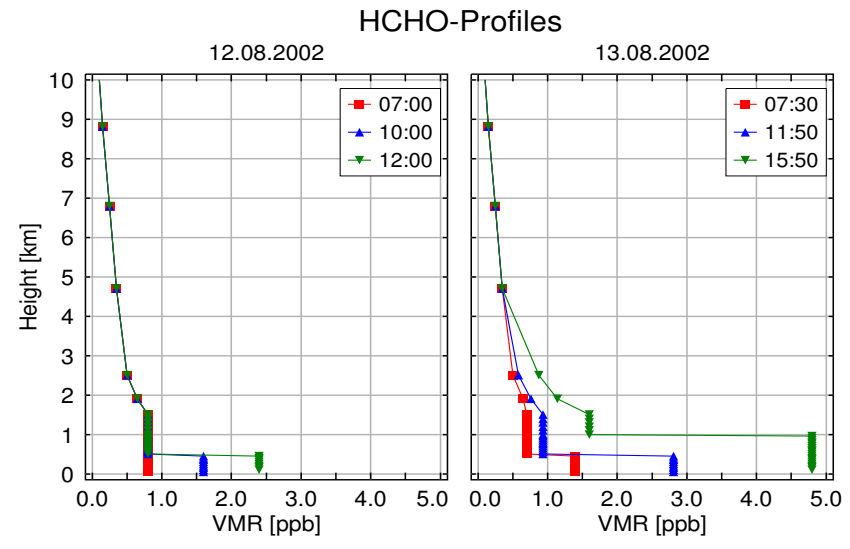

Fig. 8. Profile information retrieved for $\mathrm{HCHO}$ on 12 and 13 August from the MAX-DOAS analysis. The main sensitivity is below $5 \mathrm{~km}$ and also the main $\mathrm{HCHO}$ resides there, thus the bulk $\mathrm{HCHO}$ can be determined.

probably due to increasing aerosol extinction. Thus the observed $\mathrm{HCHO}$ increase is evidence for a change in air masses bringing $\mathrm{HCHO}$ rich and hazier air to the measurement site.

\subsection{Vertical columns}

In Fig. 7, the HCHO slant columns have been converted to vertical columns. For clarity, only one set of AMFs has been used per day. As described earlier, this is not appropriate and leads to differences between the different viewing directions when the actual profile differs from the one assumed in the AMF calculation. Obviously, profile changes on 13 August are minor so that one profile shape leads to reasonably good matching for almost the complete day (Fig. 7). For detailed analysis and to derive corresponding VMR of the ground layer two additional profiles where adjusted, which are also shown in Fig. 8. The large increase of HCHO during the afternoon is observed by all viewing directions at the same time, indicating that the vertical distribution did not change significantly during this change in air masses.

\subsection{Profiles}

Measurements from 12 August and 14 August show a more typical behaviour, where a single profile is not appropriate for all times of the day. The data of 12 August show large changes in the boundary layer (Fig. 7). The applied AMFs give consistent results for 8:00 to 10:00 UTC and again around 16:30 UTC (highlighted in yellow in Fig. 7). In the morning, data are sorted by elevation starting with the lowest one. This implies that the a priori profile contains too much HCHO in the lower layers, compared with reality. At noon the situation has changed and to match the different directions more $\mathrm{HCHO}$ in the low layers of the model profile is needed. Since the peak is mostly seen by the lower-most direction, and no according changes in the $\mathrm{O}_{4}$ or other trace

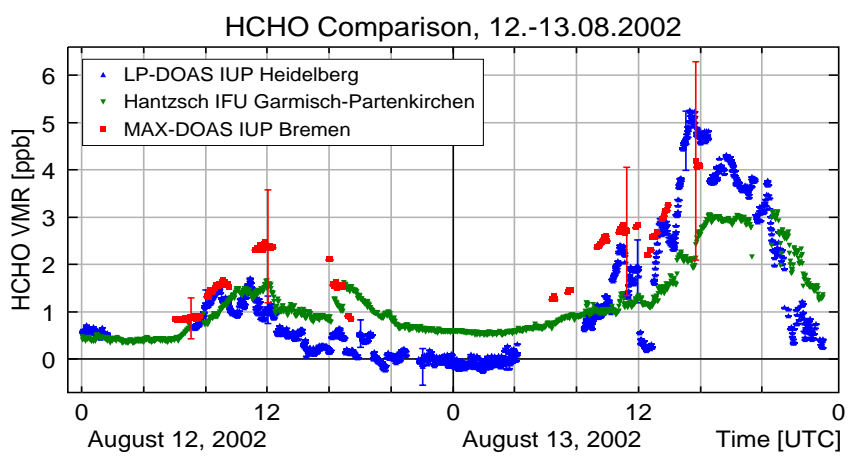

Fig. 9. Intercomparison plot showing data of a Hantzsch and a LPDOAS instrument in contrast to the $\mathrm{HCHO}$ mixing ratio derived for a atmospheric bottom layer of $500 \mathrm{~m}$ height from the MAX-DOAS analysis.

gases occur, this event has to be a local or regional emission of HCHO. In Fig. 8 an example of derived HCHO profiles for 12 August is shown. The VCDs presented in Fig. 7 correspond to the second profile, denoted by 10:00 UTC. The other two profiles were derived for the times given in the figure. The VMR of the ground layer are used in the comparison plot in the next section (Fig. 9).

For 14 August the chosen AMFs are appropriate during late afternoon, while early in the morning the different viewing directions are again ordered by elevation, this time indicating an overestimation of boundary layer $\mathrm{HCHO}$ in the a priori profile. Before noon, $\mathrm{HCHO}$ columns increase systematically, however, not at the same time for all directions. This behaviour leads us to the assumption, that there was a horizontal inhomogeneity in the HCHO field. In detail, a low layer with higher HCHO loading that is moving towards the instrument could explain the observations. It is seen first by the lower-most viewing direction, and then moving into the field of view of the other lines of sight. As mentioned the analysis is based on a homogeneous atmosphere and therefore this kind of situation cannot be treated quantitatively with the current approach if no external information is available.

\section{Comparison with independent measurements}

Finally, an attempt of a comparison with other HCHO measurements shall be made. For this purpose, data of a Hantzsch and a Long-Path (LP-DOAS) instrument operated at the same location during the campaign are available. Both techniques are well proven, for measuring formaldehyde. However, the data from the two instruments cannot be easily compared with the results of a scattered light DOAS instrument, as the air volume probed is significantly different. This problem will be discussed below. 
The Hantzsch technique is based on wet chemical fluorimetric detection, a fast and sensitive technique. The ambient air is sampled with 1 SLPM (standard liter per minute) through a $1 / 4^{\prime \prime}$ Teflon inlet line at about $3 \mathrm{~m}$ above ground and fed into the instrument. This sampling line is protected from aerosols by a nuclepore in-line filter with $0.5 \mu \mathrm{m}$ pore size to prevent degradation of the $\mathrm{HCHO}$ transfer in the polluted air in the Po-Valley. In the instrument $\mathrm{HCHO}$ is stripped from the air into the liquid phase $(0.42 \mathrm{ml} / \mathrm{min})$ in a temperature stabilised stripping coil. After conversion in a fluorescent substance in the liquid phase $\mathrm{HCHO}$ is detected by fluorimetry. The detection limit achieved is below $30 \mathrm{ppt}$ in the laboratory, for field applications less than $50 \mathrm{ppt}$. The time resolution is $90 \mathrm{~s}$ with a delay time due to the necessary chemical reaction of $4 \mathrm{~min}$. The technique was adopted from Kelly and Fortune (1994) and subsequently improved in detection limits and detection speed. The Hantzsch technique provides fast direct measurements of $\mathrm{HCHO}$ mixing ratios at one single point. Hantzsch instruments were installed at all the three ground stations as well as on-board a small research aircraft.

The second data set is provided by a long path LP-DOAS instrument (Platt, 1994). Both, LP-DOAS and MAX-DOAS are based on the DOAS technique.

The long-path telescope consists of a coaxial Newtonian telescope with transmitting and receiving optic combined in one device (for details see Geyer, 2000). In contrast to the MAX-DOAS technique, it is an active DOAS which uses an artificial light source. Light emitted by a xenon lamp passes through the open atmosphere (via the telescope). A retro reflector array in a distance of several kilometres (here $2.2 \mathrm{~km}$, $40 \mathrm{~m}$ above the ground) is used to reflect the light beam exactly back into the telescope, where it is focused onto a quartz fibre. The quartz fibre transmits the light into the spectrograph.

The instrument was placed at ground level $(1.5 \mathrm{~m})$ in close vicinity of the MAX-DOAS instrument, but with a light-path directed westward and running between 2 and $40 \mathrm{~m}$ above the ground. In contrast to the MAX-DOAS, the light path is well known and $\mathrm{HCHO}$ column densities averaged along the light path are easily derived by applying Beer's law. In order to get mixing ratios the assumption of a homogeneous air mass has to be made, which usually is appropriate.

The MAX-DOAS instrument has an open light path and in case of very good visibility the lowest viewing directions achieve light paths of up to $40 \mathrm{~km}$ within a boundary layer of $2 \mathrm{~km}$ height. As a result, the measurements are averaging over a much larger volume, and in the case of horizontal inhomogeneities, the results will differ from those of the other two instruments. Also the MAX-DOAS is integrating in the vertical direction, and even after the profile adjustment, the vertical resolution of the measurement is $500 \mathrm{~m}$ at best, and thus even the lowest point of the retrieved profile cannot directly be compared with the surface measurements.
The results of the comparison are shown in Fig. 9. The data points of the MAX-DOAS retrieval represent mixing ratios corresponding to a well mixed, homogeneous atmospheric bottom layer of $500 \mathrm{~m}$ height. For each of the highlighted areas in Fig. 7 one profile was retrieved, assuming no major changes in the aerosol and in the shape of the trace gas profile during these time periods. The VMR values within these periods result from scaling of the a priori trace gas profile to match the measured vertical column. The discontinuities seen in Fig. 9 show the limits of this approach to use one set of AMFs valid for one data point for a larger time period. As can be seen, both the absolute values and the variability is in good agreement with the two other measurements. In general, the MAX-DOAS measurements are larger than those of the Hantzsch, and often also larger than those of the LP-DOAS, although that is not the case in the evening of 13 August. Overall, the deviations between Hantzsch and MAX-DOAS are comparable to those between the other two instruments, and probably indication of the real differences between the HCHO concentrations in the different volumes. Given the large uncertainties introduced by the different measurement volumes and the choice of thickness of the lowermost layer, the agreement is judged to be excellent, and an encouraging validation of the MAX-DOAS formaldehyde retrieval.

\section{Error discussion}

When discussing the major error sources, systematic errors have to be distinguished from random errors. Systematic errors are mainly introduced by the uncertainty in the absorption cross-section of $\mathrm{HCHO}$ (quoted to be 5\% Meller and Moortgat, 2000), and possibly also by assumptions made in the radiative transfer model (see Wittrock et al., 2004, for a discussion of some of these uncertainties). The random errors shall be discussed in more detail. For this, the same steps are followed as in the analysis: first, the errors for the DSCD are analysed, then those of the tropospheric VCD and finally the uncertainties in the profile retrieval.

By looking at the noise on the DSCD, an average value of $5.6 \cdot 10^{15} \mathrm{molec} / \mathrm{cm}^{2}$ for the absolute measurement error can be estimated during the measurement campaign, mainly as a result of photon noise and detector characteristics. In case of a typical DSCD of $60 \cdot 10^{15} \mathrm{molec} / \mathrm{cm}^{2}$ this corresponds to a relative error of $9 \%$. It is possible to reduce this error further by averaging over longer time periods than the $5 \mathrm{~min}$ utes used in this study, but this will reduce temporal resolution. Also, the assumption of measuring the same atmospheric state with all viewing directions could then become less valid.

The next step, the conversion of slant into vertical columns introduces an additional error source, the AMFs. As the exact values of the AMFs depend on a multitude of factors, this step potentially introduces large uncertainties. However, 
since there are data from different viewing directions, which should give the same results, the error range for VCs becomes much smaller than for measurements using only one viewing direction. This is a result of the different sensitivity of the viewing directions to the model parameters that facilitates iterative determination of the most important quantities from the measurements themselves. Also, the highest elevation $\left(18^{\circ}\right)$ proves to be rather insensitive to changes in profile, aerosol extinction and other parameters. The uncertainties of the VCDs due to errors in the radiative transfer calculations are estimated to be $15 \%$.

The final part is the profile and mixing ratio retrieval. While the relative profile shape retrieval works quite well for the reasons given above, deriving surface mixing ratios suffers from large uncertainties. In particular, the estimate used for the well-mixed boundary layer height is critical, and thus the derived values are probably uncertain within a factor of two. A more quantitative error discussion for the retrieved mixing ratios needs the application of a full inversion for example based on the optimal estimation method. Since this was not the case for these first results presented within this article, the error treatment is necessarily limited to a more qualitative discussion. As already discussed in Sect. 4, horizontal inhomogeneities can have a large impact on the differences observed in the different viewing directions. This can lead to inconsistent and even unphysical vertical profiles if an inversion is attempted that is not accounting for these gradients. Similarly, clouds can change the radiative transfer in many ways, and although the effects could be modelled in principle, such data has in practice to be excluded from the analysis.

\section{Conclusions}

During the first FORMAT campaign in July/August 2002, measurements with a multi-mxis DOAS instrument were performed for 30 days. The measurements were analysed for $\mathrm{HCHO}$, and results for three selected days are presented. By using not only the zenith sky observations, but also measurements pointed at the horizon, a high sensitivity to tropospheric $\mathrm{HCHO}$ could be obtained, and also information on its vertical distribution. This is achieved by iterating the radiative transfer calculations until agreement is found for the measurements from all viewing directions. Three different HCHO measurement quantities were retrieved from the data: The tropospheric vertical column, a tropospheric profile and a mixing ratio close to the ground.

The first quantity can be retrieved with high accuracy (better than $1.5 \cdot 10^{15}$ molec $/ \mathrm{cm}^{2}$ ), mainly due to the fact that the DOAS retrieval is very sensitive and the radiative transfer in the $18^{\circ}$ viewing direction simple and not depending much on the assumptions made for the vertical profile of the absorber or the aerosol extinction. The tropospheric column is a measure of the total amount of $\mathrm{HCHO}$ present in the lower atmosphere, and well suited for validation of measurements from satellites such as GOME or SCIAMACHY.

The vertical profile retrieved from the differences observed in the five viewing directions has a much larger uncertainty and also a rather coarse vertical resolution. It is however quite sensitive to changes in the lowest two kilometres, and can give an estimate of where the bulk of the HCHO is located. In combination with the tropospheric column, this is very valuable information, in particular if the relative impact of transport and local emissions is to be studied.

The last quantity, mixing ratios close to the surface has the largest uncertainty, in particular as a height has to be assumed for the well mixed boundary layer, and this assumption directly translates into the number retrieved for the mixing ratio. It therefore should not be considered as the main output quantity from the MAX-DOAS measurements.

A comparison of the mixing ratios derived from the MAXDOAS spectra with independent in-situ measurements from a Hantzsch and a long path DOAS system has also been performed. The agreement is excellent and well within the error bars for both in the absolute values and the diurnal variation. This comparison provides a first validation for the retrieval method, although clearly the air sampled by the three instruments was not identical.

A crucial input for the radiative transfer calculation is the aerosol extinction profile that can be retrieved from the data by analysing the measurements of the oxygen dimer $\mathrm{O}_{4}$. Again, the results from all viewing directions are compared and the extinction profile used iterated until closure is achieved. In the case study presented here, the aerosol extinction as retrieved from the measurements varied from $0.05 \mathrm{~km}^{-1}$ to $0.5 \mathrm{~km}^{-1}$, and only by incorporating the aerosol retrieval in the data analysis, meaningful $\mathrm{HCHO}$ profiles could be derived. The results also indicate, that MAXDOAS measurements might be used to provide a height resolved aerosol extinction profile, a quantity that is difficult to measure with other methods.

For the presented days we derived vertical columns of $\mathrm{HCHO}$ between 6 and $18 \cdot 10^{15} \mathrm{molec} / \mathrm{cm}^{2}$. Different sources for the observed diurnal variations of the vertical columns (Fig. 7) were identified from our analysis. First, a typical pattern of local formaldehyde emission was seen on 12 August. Second, a major change in the airmass could be observed showing a strong increase in $\mathrm{HCHO}$ and aerosol (according the changes of $\mathrm{O}_{4}$ ). The analysis of the temporal variation of the measurements of different viewing geometries also revealed that the transport of inhomogeneities (plumes) of $\mathrm{HCHO}$ can also be seen in the measurements.

In summary, it has been shown MAX-DOAS measurements can provide tropospheric columns and some profile information on HCHO in polluted areas with an automated and relatively simple instrument. The data can also be used to derive information on other absorbers such as $\mathrm{O}_{3}$ and $\mathrm{NO}_{2}$, and also on aerosol extinction. Analysis of the full time series from the first and also the second FORMAT campaign 
(September 2003) is under way and is expected to provide more information on pollution levels and the transport and formation of photo-oxidants in the Milan area.

Acknowledgements. The authors would like to thank the organisers of the FORMAT campaign and in particular B. Larsen, now at JRC in Ispra (Italy), for their great support before and during the measurements. The analysis of the local meteorologic conditions was supported by measurements provided by C. Ordóñez from Paul Scherrer Institute in Villigen (Switzerland).

In addition we acknowledge the helpful comments by $\mathrm{T}$. Wagner (IUP, Uni Heidelberg, Germany). Parts of this work have been funded by the European Union (contract FORMAT, EVK2-200100085) and the University of Bremen.

Edited by: A. Hofzumahaus

\section{References}

Bobrowski, N., Hönninger, G., Galle, B., and Platt, U.: Detection of bromine monoxid in a volcanic plume, Nature, 423, 273-276, doi:10.1038/nature01625, 2003.

Bovensmann, H., Burrows, J. P., Buchwitz, M., Frerick, J., Noel, S., Rozanov, V. V., Chance, K. V., and Goede, A. P. H.: SCIAMACHY: Mission Objectives and Measurement Modes, J. Atmos. Sci., 56, 127-150, 1999.

Burrows, J. P., Weber, M., Buchwitz, M., Ladstätter-Weißenmayer, A., Richter, A., DeBeek, R., Hoogen, R., Bramstedt, K., Eichmann, K.-U., Eisinger, M., and Perner, D.: The Global Ozone Monitoring Experiment (GOME): Mission concept and first scientific results, J. Atmos. Sci., 56, 151-175, 1999.

Dieke, G. H. and Kistiakowsky, G. B.: The Structure of the Ultraviolet Absorption Spectrum of Formaldehyde I, Phys. Rev., 45, 4-28, 1934.

Finlayson-Pitts, B. J. and Pitts, J. N.: Upper and Lower Atmosphere, Academic Press, San Diego, 2000.

Geyer, A.: The Role of the Nitrate Radical in the Boundary Layer - Observations and Modeling Studies, Dissertation, Universität Heidelberg, 2000.

Grainger, J. F. and Ring, J.: Anomalous Fraunhofer line profiles, Nature, 193, 762, 1962.

Hönninger, G. and Platt, U.: The role of $\mathrm{BrO}$ and its vertical distribution during surface ozone depletion at Alert, Atmos. Environ., 36, 2481-2489, doi:10.1016/S1352-2310(02)00104-8, 2002.

Kelly, T. J. and Fortune, C. R.: Continuous Monitoring of Gaseous Formaldehyde using an improved Fluorescent Approach, Int. J. Environ. Anal. Chem., 54, 249-263, 1994.

Leser, H., Hönninger, G., and Platt, U.: MAX-DOAS measurements of $\mathrm{BrO}$ and $\mathrm{NO}_{2}$ in the marine boundary layer, Geophys. Res. Lett., 30, 1537, doi:10.1029/2002GL015811, 2003.
Meller, R. and Moortgat, G. K.: Temperature dependence of the absorption cross sections of formaldehyde between 223 and 323 $\mathrm{K}$ in the wavelength range $225-375 \mathrm{~nm}$, J. Geophys. Res., 105, 7089-7101, 2000.

Palmer, P. I., Jacob, D. J., Chance, K., Martin, R. V., Spurr, R. J. D., Kurosu, T. P., Bey, I., Yantosca, R., Fiore, A., and Li, Q.: Air mass factor formulation for spectroscopic measurements from satellites: Application to formaldehyde retrievals from the Global Ozone Monitoring Experiment, J. Geophys. Res., 106, 14 539-14 550, 2001.

Platt, U.: Differential optical absorption spectroscopyy (DOAS), in: Air Monitoring by Spectroscopic Techniques, edited by: Sigrist, M. W., vol. 127, Chem. Anal. Ser., John Wiley, New York, 1994.

Prévôt, A. S. H., Staehelin, J., Kok, G. L., Schillawski, R. D., Neinigner, B., Staffelbach, T., Neftel, A., Wernli, H., and Dommen, J.: The Milan photooxidant plume, J. Geophys. Res., 102, 23 375-23 388, 1997.

Roozendael, M. v., Fayt, C., Post, P., Hermans, C., and Lambert, J.C.: Retrieval of $\mathrm{BrO}$ and $\mathrm{NO}_{2}$ from UV-Visible Observations, in: Sounding the troposphere from space: a new era of atmospheric chemistry, edited by: Borell, P., Burrows, J. P., and Platt, U., Springer, ISBN 3-540-40873-8, 2003.

Rozanov, A. V., Rozanov, V. V., and Burrows, J. P.: A Numerical Radiative Transfer Model for a Spherical Planetary Atmosphere: Combined Differential-Integral Approach involving the Picard Iterativ Approximation, Journal of Quantitative Spectroscopy and Radiative Transfer, 69, 491-512, doi:10.1016/S0022-4073(00)00100-X, 2001.

Solomon, S., Schmeltekopf, A. L., and Sanders, R. W.: On the Interpretation of Zenith Sky Absorbtion Measurements, J. Geophys. Res., 92, 8311-8319, 1987.

Vountas, M., Rozanov, V. V., and Burrows, J. P.: Ring Effect: Impact of Rotational Raman Scattering on Radiative Transfer in Earth's Atmosphere, Journal of Quantitative Spectroscopy and Radiative Transfer, 60, 943-961, 1998.

Wagner, T., Friedeburg, C. v., Wenig, M., Otten, C., and Platt, U.: UV-visible observations of atmospheric $\mathrm{O}_{4}$ absorptions using direct moonlight and zenith-scattered sunlight for clearsky and cloudy sky conditions, J. Geophys. Res., 107, 4424, doi:10.1029/2001JD001026, 2002.

Weber, R. O. and Prévôt, A. S. H.: Climatology of ozone transport from the free troposphere into the boundary layer south of the Alps during North Foehn, J. Geophys. Res., 107, 4030, doi:10.1029/2001JD000987, 2002.

Wittrock, F., Oetjen, H., Richter, A., Fietkau, S., Medeke, T., Rozanov, A., and Burrows, J. P.: MAX-DOAS measurements of atmospheric trace gases in Ny-Ålesund, Atmos. Chem. Phys., 4, 955-966, 2004,

SRef-ID: 1680-7324/acp/2004-4-955. 